\title{
BMJ Open Predicting mortality in adults with suspected infection in a Rwandan hospital: an evaluation of the adapted MEWS, qSOFA and UVA scores
}

\author{
Amanda Klinger, ${ }^{1,2}$ Ariel Mueller, ${ }^{2,3}$ Tori Sutherland, ${ }^{2,3}$ Christophe Mpirimbanyi,, ${ }^{4,5}$ \\ Elie Nziyomaze, ${ }^{4,5}$ Jean-Paul Niyomugabo, ${ }^{5}$ Zack Niyonsenga, ${ }^{5}$ Jennifer Rickard, ${ }^{4,6}$ \\ Daniel S Talmor, ${ }^{2,3}$ Elisabeth Riviello (1) 2,7
}

To cite: Klinger A, Mueller A, Sutherland T, et al. Predicting mortality in adults with suspected infection in a Rwandan hospital: an evaluation of the adapted MEWS, qSOFA and UVA scores. BMJ Open 2021;11:e040361. doi:10.1136/ bmjopen-2020-040361

- Prepublication history and additional materials for this paper is available online. To view these files, please visit the journal online (http://dx.doi. org/10.1136/bmjopen-2020040361).

Received 12 May 2020

Revised 20 November 2020

Accepted 30 November 2020

Check for updates

(c) Author(s) (or their employer(s)) 2021. Re-use permitted under CC BY-NC. No commercial re-use. See rights and permissions. Published by BMJ.

For numbered affiliations see end of article.

Correspondence to

Dr Elisabeth Riviello;

beth_riviello@post.harvard.edu

\section{ABSTRACT}

Rationale Mortality prediction scores are increasingly being evaluated in low and middle income countries (LMICs) for research comparisons, quality improvement and clinical decision-making. The modified early warning score (MEWS), quick Sequential (Sepsis-Related) Organ Failure Assessment (qSOFA), and Universal Vital Assessment (UVA) score use variables that are feasible to obtain, and have demonstrated potential to predict mortality in LMIC cohorts.

Objective To determine the predictive capacity of adapted MEWS, qSOFA and UVA in a Rwandan hospital.

Design, setting, participants and outcome measures We prospectively collected data on all adult patients admitted to a tertiary hospital in Rwanda with suspected infection over 7 months. We calculated an adapted MEWS, qSOFA and UVA score for each participant. The predictive capacity of each score was assessed including sensitivity, specificity, positive and negative predictive value, $\mathrm{OR}$, area under the receiver operating curve (AUROC) and performance by underlying risk quartile. Results We screened 19178 patient days, and enrolled 647 unique patients. Median age was 35 years, and in-hospital mortality was $18.1 \%$. The proportion of data missing for each variable ranged from $0 \%$ to $11.7 \%$. The sensitivities and specificities of the scores were: adapted MEWS $>4,50.4 \%$ and $74.9 \%$, respectively; qSOFA $>2,24.8 \%$ and $90.4 \%$, respectively; and UVA $>4$, $28.2 \%$ and $91.1 \%$, respectively. The scores as continuous variables demonstrated the following AUROCs: adapted MEWS 0.69 (95\% Cl 0.64 to 0.74$)$, qSOFA $0.65(95 \% \mathrm{Cl}$ 0.60 to 0.70$)$, and UVA $0.71(95 \% \mathrm{Cl} 0.66$ to 0.76$)$; there was no statistically significant difference between the discriminative capacities of the scores.

Conclusion Three scores demonstrated a modest ability to predict mortality in a prospective study of inpatients with suspected infection at a Rwandan tertiary hospital. Careful consideration must be given to their adequacy before using them in research comparisons, quality improvement or clinical decision-making.

\section{INTRODUCTION}

Multiple mortality prediction models have been developed or validated in low and
Strengths and limitations of this study

- We evaluated the three severity of illness (SOI) scores in the literature that are most likely to be feasible and predictive in low and middle income countries (LMIC) settings; this includes the first hospital-wide evaluation of Universal Vital Assessment score, the only score that was developed using LMIC cohorts.

- Many SOI scores are developed and tested intheintensive care unit (ICU) populations while our analysis also includes hospitalised patients outside the ICU; this is important because many critically ill patients in LMICs remain outside the ICU due to resource constraints.

- We analysed the predictive capacity of the SOI models as both continuous and dichotomous scores and using multiple metrics, including sensitivity, specificity, positive and negative predictive value, $\mathrm{OR}$, area under the receiver operating curve and performance by underlying risk quartile.

- Vital signs used in the scores were collected at different times in the participants' hospitalisations, depending on how they met inclusion criteria for the study (time of fever, operation or culture sample retrieval); while this may decrease the predictive capacity of the scores, it also mirrors how the scores might be used in practice.

- The results from this single-centre study among adults with suspected infection may not be generalisable to other populations; this variability in predictive capacity is a known challenge in using SOl scores and the reason it is important to validate a score in a particular site before using it.

middle income countries (LMICs) over the last 5 years. ${ }^{1-11}$ The proposed uses of these models include identifying patients at acute risk for deterioration in order to trigger increased levels of care, ${ }^{311-15}$ more informed allocation of scarce resources, ${ }^{13} 15$ benchmarking for quality assessment and quality improvement ${ }^{1}$ and controlling for severity of illness (SOI) in future trials. ${ }^{131617}$ In addition, 
updates to definitions of critical illness syndromes, most notably sepsis and acute respiratory distress syndrome, have increasingly emphasised definitions that have predictive validity. ${ }^{18} 19$

The modified early warning score (MEWS) was first reported describing 709 medical patients in a district hospital in the UK in 2001, ${ }^{20}$ and was based on an early warning score developed and published in an abstract in $1997 .^{21}$ It was created by assigning weighted scores to each vital sign based on severity of the vital sign abnormality, and it has since been tested in multiple LMIC sites. ${ }^{8} 122223$ The quick Sequential (Sepsis-Related) Organ Failure Assessment (qSOFA) score was developed as part of an international re-defining of sepsis, using high income country (HIC) hospital administrative data ${ }^{19}$ and retrospectively tested in nine sites in LMICs; it demonstrated variable predictive capability across these sites. ${ }^{15}$ qSOFA was also prospectively tested in a study from an upper middle income country with multiple sites. ${ }^{11}$ The Universal Vital Assessment (UVA) score was recently developed using linear regression in 15 in-hospital cohorts from 6 African countries, and showed good predictive capability across the entire derivation population, with no reporting on its performance in the individual cohorts. ${ }^{13}$ It has only been assessed in one small emergency department cohort outside the initial derivation population. ${ }^{23}$

All three scores use accessible bedside clinical measures and are, therefore, appealing for LMIC settings where laboratory values and detailed comorbidity histories are often not available. All three scores have also been developed for hospital ward patients, which is relevant to LMICs, where critically ill patients often remain in general wards due to the scarcity of intensive care unit (ICU) beds.

We prospectively collected data on all adult hospitalised patients with suspected infection over a 7-month period in a study of antimicrobial resistance patterns in a tertiary referral hospital in Rwanda. ${ }^{24}$ The current study was planned as part of the original study design, and is a secondary analysis of this data evaluating the predictive capacity of adapted MEWS, qSOFA and UVA scores for in-hospital mortality in this population.

\section{METHODS}

\section{Study oversight}

The Institutional Review Board of the University of Rwanda, College of Medicine and Health Sciences in Kigali, Rwanda and the Committee on Clinical Investigations at Beth Israel Deaconess Medical Center (BIDMC) in Boston, Massachusetts approved the study. Verbal consent for participation was obtained using a script in the participant's primary language.

\section{Patient and public involvement}

This research was performed without explicit patient feedback on the design or implementation. Results will be available to the public through open access publication.

\section{Setting}

The study took place at the University Teaching Hospital of Kigali. The hospital is a public academic tertiary referral hospital in Kigali, Rwanda. It is one of three public referral hospitals in a country of approximately 12 million people, with 560 total beds including a 35-bed adult emergency department, a 7-bed ICU, a 4-bed stepdown unit and approximately 12000 admissions each year.

\section{Inclusion criteria and data collection}

We prospectively enrolled all hospitalised adult patients (age $\geq 15$ years, the hospital's cut-off for adult hospital ward admission) with suspected infection between 25 January 2017 and 14 August 2017 as part of a study examining antimicrobial resistance patterns. ${ }^{24}$ All hospitalised patients were screened for inclusion criteria each day of their hospitalisation. We recorded the number of patients screened each day in each area of the hospital; we did not record the number of unique patients screened over the entire study period. Patients met inclusion criteria if they had temperature $\leq 35.0^{\circ} \mathrm{C}$ or $\geq 38.0^{\circ} \mathrm{C}$ and suspected infection, underwent surgery for an infectious process or had a positive microbial culture collected by the clinical team. For those who met inclusion criteria and provided consent, demographic and clinical data needed for each of the scores were collected at one time point from each participant's chart by study research assistants. Vital sign and mental status data to include in the models were collected at the time of fever or hypothermia, the time of surgery or the time of culture sample collection, depending on the inclusion criteria met for each participant. For patients who met more than one inclusion criteria, the time point for clinical data collection was based on the first inclusion criteria met. Participants were followed through hospital discharge to determine length of stay and in-hospital mortality. All coded data were entered into a secure online database, REDCap (Vanderbilt University, Nashville, Tennessee), which was hosted by BIDMC.

\section{Definitions}

MEWS includes five variables, with scores between 0 and 3 assigned for each variable ${ }^{20}$ table 1 . It yields a maximum score of 14 , with a score $>4$ considered to be high risk for mortality in prior studies. ${ }^{20}$ Because we collected altered mental status as a binary variable (present or not), we adapted this variable in the MEWS score to be 0 for normal mental status and 2 for any altered mental status, rather than a range of severity of altered mental statuses from 0 to 3 . qSOFA includes three variables, with 1 point given to each abnormal value, a maximum score of 3 and $\geq 2$ considered high risk. ${ }^{15}$ UVA includes seven variables, with variable points given for each abnormality. It yields a maximum score of 13 , with $>4$ considered high risk based on its derivation study. ${ }^{13}$

To replicate the methods for predictive validity in the original qSOFA and qSOFA LMIC validation studies, ${ }^{15} 25$ 
Table 1 Variables and values in adapted MEWS, qSOFA and UVA scores

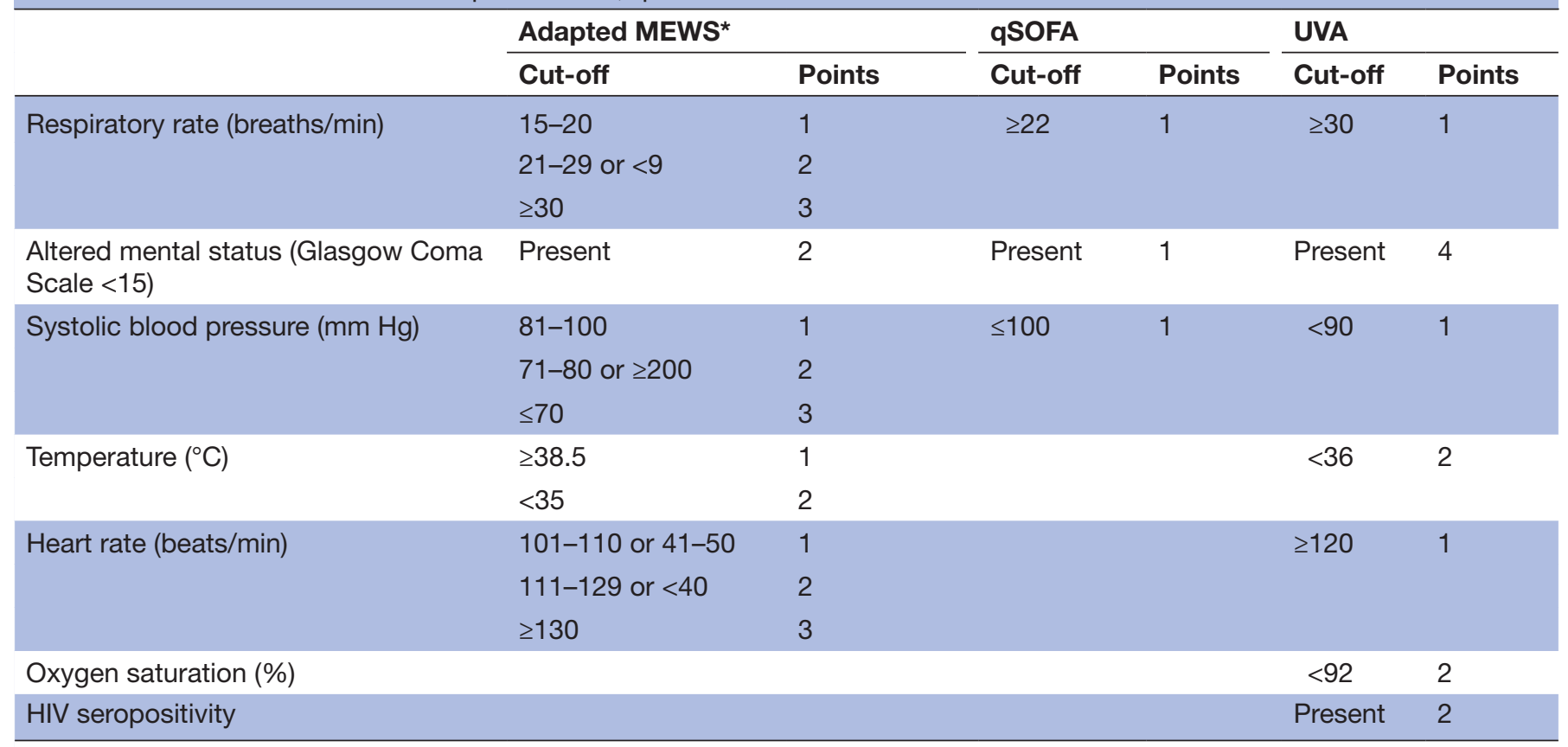

${ }^{*}$ The adaptation to the MEWS score pertains to the altered mental status score. In the original MEWS, 0 point was assigned for alert patients, 1 if they reacted to voice, 2 if they reacted to pain and 3 if they were unresponsive. In our adapted MEWS, we assign 0 point for an alert patient and 2 for a patient with any altered mental status.

GCS, Glasgow Coma Scale; MEWS, modified early warning score ; qSOFA, quick Sequential (Sepsis-Related) Organ Failure Assessment; UVA, Universal Vital Assessment.

we also calculated a baseline risk model to stratify the population, using the same variables used in these studies: age, sex, HIV status and hospital transfer status (whether the patient had been transferred from another facility).

\section{Data analysis}

The primary outcome of interest was in-hospital mortality. The sample size was determined based on adequate power for the antimicrobial resistance study from which this cohort was taken, and is described in the methods of that study. ${ }^{24}$ Adapted MEWS, qSOFA and UVA scores were calculated for all enrolled participants. Missing data were assumed to be within normal range, with no additional points assigned. Data are presented as median (IQR) or frequency (proportion) depending on variable type. Normality was assessed with the Shapiro-Wilk test. Demographic differences between survivors and non-survivors were assessed with a Wilcoxon rank-sum test, $\chi^{2}$ or Fisher's exact test, as appropriate. Sensitivity, specificity, positive and negative predictive values for the previously reported cut-offs for each score are reported. Separate unadjusted logistic regression models were used to generate ORs and 95\% CIs for adapted MEWS, qSOFA and UVA. Multivariable logistic regression models using the four variables noted above were calculated for the baseline risk model.

We used the predicted probabilities from our baseline risk model to stratify our results into risk quartiles, presenting ORs and 95\% CIs for adapted MEWS, qSOFA and UVA with their previously defined cut-offs separately, as was done in the original LMIC cohort
qSOFA study. ${ }^{15}$ We calculated the discriminative ability of adapted MEWS, qSOFA and UVA as continuous variables and found the area under the receiver operating characteristic (AUROC) curves for each of these models. We also calculated the discriminative ability of the three scores as continuous variables in models with baseline risk adjustment.

Data analysis was performed using SAS 9.4 (SAS Institute, Cary, North Carolina) with two-sided p values $<0.05$ considered statistically significant.

\section{RESULTS}

We screened every patient in the hospital for suspected infection each day of the study period, for a total of 19178 patient days screened. We enrolled 647 of the 648 unique patients who met our criteria for suspected infection; the only exclusion was one patient who met study criteria, but declined enrollment. Within this study population, 497 participants $(76.8 \%)$ had hypo or hyperthermia and suspected infection, 308 participants $(47.6 \%)$ underwent surgery for an infectious process, and 273 participants $(42.2 \%)$ had a positive microbial culture (online supplemental figure 1). The median age was 35 years (IQR 27-51) and 347 (53.6\%) of participants were male (table 2). Known pre-existing comorbidities were present in $143(22.1 \%)$ of participants, and $68(10.5 \%)$ of participants were known to be HIV positive. 
Open access

Table 2 Baseline characteristics of patients admitted with suspected infection

\begin{tabular}{|c|c|c|c|c|}
\hline & Total $n=647$ & Survivors $n=530$ & Non-survivors $n=117$ & $P$ value \\
\hline \multicolumn{5}{|l|}{ Demographics } \\
\hline Age, median (IQR) & $35.0(27.0,51.0)$ & $35.0(27.0,51.0)$ & $36.0(27.0,56.0)$ & 0.46 \\
\hline HIV positive, n (\%) & $68(10.5)$ & $52(9.8)$ & $16(13.7)$ & 0.22 \\
\hline Other known pre-existing co-morbidity*, n (\%) & $143(22.1)$ & $106(20.0)$ & $37(31.6)$ & 0.01 \\
\hline \multicolumn{5}{|l|}{ Adapted† MEWS components } \\
\hline Respiratory rate, beats/min & & & & 0.0002 \\
\hline $9-14$ & $72(11.1)$ & $51(9.6)$ & $21(17.9)$ & \\
\hline $15-20$ & $417(64.4)$ & $361(68.1)$ & $56(47.9)$ & \\
\hline Systolic blood pressure, $\mathrm{mm} \mathrm{Hg}$ & & & & 0.13 \\
\hline $100-199$ & $533(82.4)$ & $437(82.4)$ & $96(82.0)$ & \\
\hline $81-100$ & $97(15.0)$ & $81(15.3)$ & $16(13.7)$ & \\
\hline $71-80$ or $\geq 200$ & $12(1.8)$ & $10(1.9)$ & $2(1.7)$ & \\
\hline$\leq 70$ & $5(0.8)$ & $2(0.4)$ & $3(2.6)$ & \\
\hline Temperature & & & & 0.002 \\
\hline$\geq 38.5^{\circ} \mathrm{C}$ & $309(47.8)$ & $238(44.9)$ & $71(60.7)$ & \\
\hline $35^{\circ} \mathrm{C}-38.4^{\circ} \mathrm{C}$ & $338(52.2)$ & $292(55.1)$ & $46(39.3)$ & \\
\hline$<35^{\circ} \mathrm{C}$ & $0(0)$ & $0(0)$ & $0(0)$ & \\
\hline \multicolumn{5}{|l|}{ qSOFA components } \\
\hline Altered mental status & $150(23.2)$ & $92(17.4)$ & $58(49.6)$ & $<0.0001$ \\
\hline Systolic blood pressure $\leq 100$ & $112(17.3)$ & $91(17.2)$ & $21(17.9)$ & 0.84 \\
\hline Respiratory rate $\geq 22$ & $147(22.7)$ & $110(20.7)$ & $37(31.6)$ & 0.01 \\
\hline qSOFA $\geq 2$ & $81(12.5)$ & $52(9.8)$ & $29(24.8)$ & $<0.0001$ \\
\hline \multicolumn{5}{|l|}{ UVA components } \\
\hline Temperature $<36^{\circ} \mathrm{C}$ & $12(1.8)$ & $12(2.3)$ & $0(0)$ & 0.10 \\
\hline Heart rate $\geq 120$ & $175(27.0)$ & $129(24.3)$ & $46(39.3)$ & 0.001 \\
\hline Respiratory rate $\geq 30$ & $37(5.7)$ & $25(4.7)$ & $12(10.3)$ & 0.02 \\
\hline Systolic blood pressure $<90 \mathrm{~mm} \mathrm{Hg}$ & $37(5.7)$ & $29(5.5)$ & $8(6.8)$ & 0.56 \\
\hline Oxygen saturation $<92 \%$ & $149(23.0)$ & $118(22.3)$ & $31(26.5)$ & 0.33 \\
\hline Altered mental status & $150(23.2)$ & $92(17.4)$ & $58(49.6)$ & $<0.0001$ \\
\hline HIV positive & $68(10.5)$ & $52(9.8)$ & $16(13.7)$ & 0.22 \\
\hline UVA $>4$ & $80(12.4)$ & $47(8.9)$ & $33(28.2)$ & $<0.0001$ \\
\hline
\end{tabular}

*Includes patients who had any of the following documented co-morbidities: diabetes, hypertension, tuberculosis, cancer and/or severe malnutrition. †The adaption to the MEWS score pertains to the altered mental status score. In the original MEWS, 0 point was assigned for alert patients, 1 if they reacted to voice, 2 if they reacted to pain and 3 if they were unresponsive. In our adapted MEWS, we assign 0 point for an alert patient and 2 for a patient with any altered mental status.

MEWS, modified early warning score; qSOFA, quick Sequential (Sepsis-Related) Organ Failure Assessment; UVA, Universal Vital Assessment. 

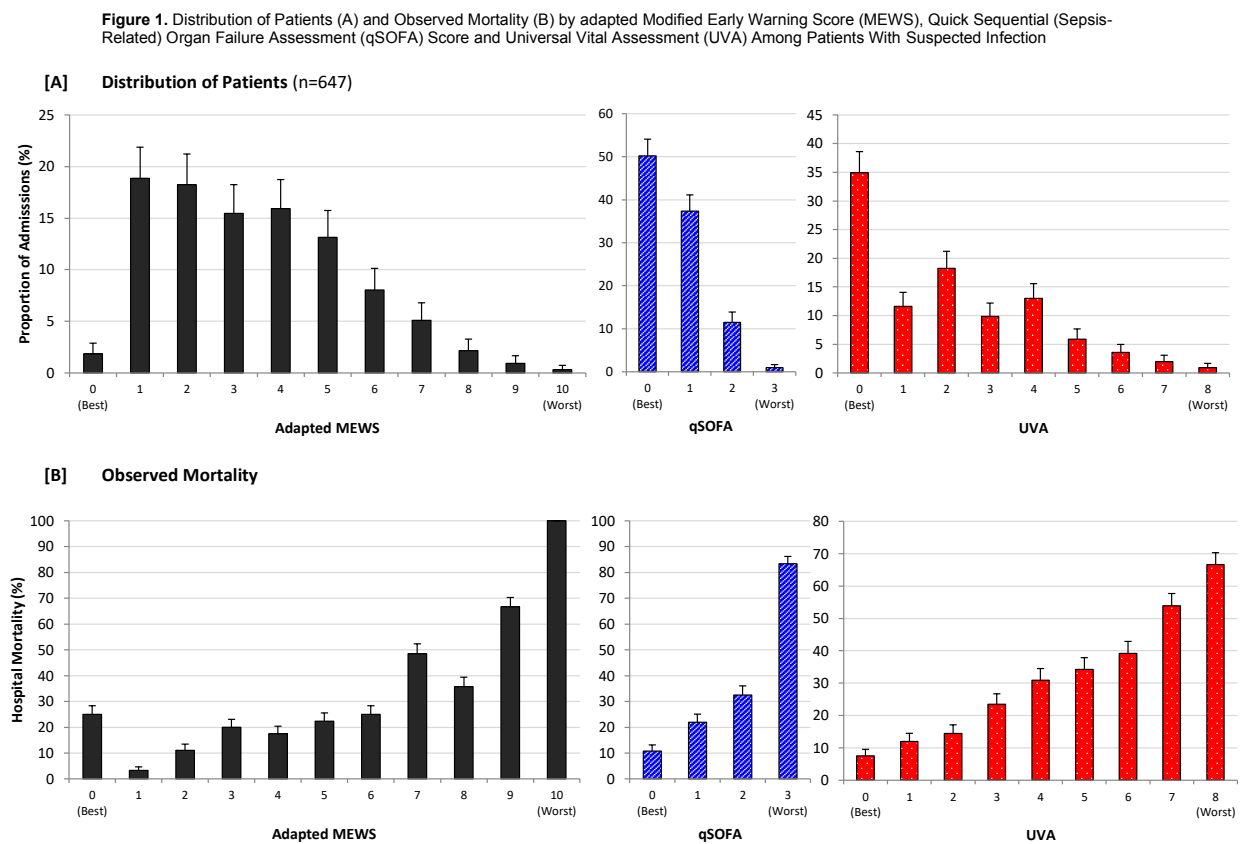

Figure 1 Distribution of patients (A) and observed mortality (B) with standard errors by adapted modified early warning score (MEWS), quick Sequential (Sepsis-Related) Organ Failure Assessment (qSOFA) score and Universal Vital Assessment (UVA) among patients with suspected infection.

In the full cohort, the in-hospital mortality rate was $18.1 \%$ (117 of 647 participants). An adapted MEWS score of $>4$ was present in $29.7 \%(192 / 647)$ of cases, qSOFA score of $\geq 2$ was present in $12.5 \%(81 / 647)$ of cases, while a UVA score $>4$ was present in $12.4 \%(80 / 647)$ of cases (table 2). The full distribution for each score is shown in figure 1, with adapted MEWS range $0-10$, median 3, IQR 2- 5; qSOFA range 0-3, median 0, IQR 0- 1; and UVA range $0-8$, median 2 , IQR $0-4$. The proportion of data that was missing for the components of the scores ranged from $0 \%$ to $11.7 \%$ (online supplemental table 1).

The sensitivity and specificity of the adapted MEWS score with cut-off value $>4$ to predict in-hospital mortality were $50.4 \%(59 / 117)$ and $74.9 \%(397 / 530)$, respectively (table 3 ). The sensitivity and specificity of qSOFA with cut-off value $\geq 2$ were $24.8 \%(29 / 117)$ and $90.4 \%$ $(479 / 530)$, respectively. For the UVA score with cutoff value $>4$, the sensitivity and specificity were $28.2 \%$ $(33 / 117)$ and $91.1 \%(483 / 530)$, respectively. The sensitivity, specificity, and positive and negative predictive values for each score using the full range of possible cutoff values are presented in online supplemental table 2. The unadjusted ORs for adapted MEWS $>4$, qSOFA $\geq 2$ and UVA $>4$ were 3.04 (95\% CI 2.01 to 4.59 ), 3.10 (95\% CI 1.86 to 5.15 ) and 4.04 (95\% CI 2.44 to 6.67 ), respectively. The OR for hospital mortality was most often $>1$ for each binary score within each quartile of baseline risk, though the 95\% CI for the OR crossed 1 for qSOFA and UVA in quartile 4, and for adapted MEWS in quartile 1 (online supplemental figure 2).

Overall, increasing scores for adapted MEWS, qSOFA and UVA corresponded with increasing mortality, though this was not true for every 1 point increase in adapted
MEWS (figure 1). For each 1 point increase in score as a continuous variable, the unadjusted ORs were: adapted MEWS 1.41 (95\% CI 1.28 to 1.56), qSOFA 2.20 (95\% CI 1.68 to 2.88 ) and UVA 1.46 (1.32 to 1.61 ) (online supplemental table 3).

The AUROC for each score as a continuous variable was: adapted MEWS 0.69 (95\% CI 0.64 to 0.74 ), qSOFA 0.65 (95\% CI 0.60 to 0.70$)$ and UVA 0.71 (95\% CI 0.66 to 0.76 ) (figure 2, online supplemental table 3 ). There

Table 3 Predictive capacity of adapted MEWS, qSOFA and UVA scores

\begin{tabular}{llll}
\hline & $\begin{array}{l}\text { Adapted } \\
\text { MEWS* }>\text { 4 }\end{array}$ & qSOFA $\geq \mathbf{2}$ & UVA >4 \\
\hline $\begin{array}{l}\text { Unadjusted } \\
\text { Sensitivity }\end{array}$ & 50.4 & 24.8 & 28.2 \\
$\begin{array}{l}\text { Specificity } \\
\begin{array}{l}\text { Positive } \\
\text { predictive } \\
\text { value }\end{array}\end{array}$ & 74.9 & 90.4 & 91.1 \\
$\begin{array}{l}\text { Negative } \\
\text { predictive } \\
\text { value }\end{array}$ & 87.2 & 36.2 & 41.2 \\
$\begin{array}{l}\text { OR (95\% Cl) } \\
\text { OR.04 (2.01 to } 3.10 \text { (1.86 to 5.15) }\end{array}$ & 4.04 (2.44 to 6.67) \\
\hline
\end{tabular}

${ }^{*}$ The adaptation to the MEWS score pertains to the altered mental status score. In the original MEWS, 0 point was assigned for alert patients, 1 if they reacted to voice, 2 if they reacted to pain, and three if they were unresponsive. In our adapted MEWS, we assign 0 point for an alert patient and 2 for a patient with any altered mental status. MEWS, modified early warning score; qSOFA, quick Sequential (Sepsis-Related) Organ Failure Assessment; UVA, Universal Vital Assessment. 


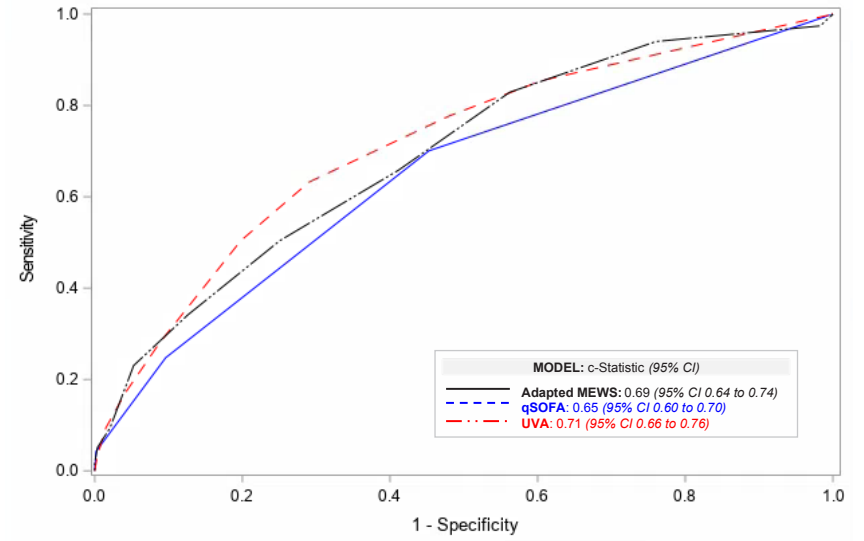

Figure 2 Receiver operating characteristic curves for adapted MEWS, qSOFA or UVA criteria as continuous variables. MEWS, modified early warning score; qSOFA, quick Sequential (Sepsis-Related) Organ Failure Assessment; UVA, Universal Vital Assessment.

was no statistically significant difference between the AUROCs for the three scores as pairwise comparisons: UVA versus adapted MEWS $p=0.57$; UVA versus $q S O F A$ $\mathrm{p}=0.09$; and adapted MEWS versus qSOFA $\mathrm{p}=0.26$.

The AUROC for the baseline risk model was $0.57(95 \%$ CI 0.52 to 0.63$)$. Adding adapted MEWS, qSOFA and UVA as continuous variables to the baseline risk model changed the AUROC to 0.72 (95\% CI 0.66 to 0.77 ), 0.68 (95\% CI 0.63 to 0.74 ), and 0.72 (95\% CI 0.66 to 0.77 ), respectively (online supplemental figure 3 , table 4 ).

\section{DISCUSSION}

In a prospective study of 647 patients with suspected infection in a Rwandan tertiary referral hospital, we found that the adapted MEWS, qSOFA and UVA scores had modest ability to predict mortality. Using previously defined cut-offs for each of the scores, adapted MEWS had sensitivity and specificity of $50 \%$ and $75 \%$, respectively, while qSOFA and UVA were less sensitive, but had higher specificity $(25 \%$ and $90 \%$, respectively, for qSOFA and $28 \%$ and $91 \%$, respectively, for UVA). AUROCs for the continuous scores ranged from 0.65 to 0.71 , with no AUROC for continuous score demonstrating statistically significant superiority to another.

We presented the performance of the three scores using the continuous scores, continuous scores in addition to a baseline risk model, and binary scores using previously defined cut-off values. Depending on the intended use of the scores, any of these might be appropriate in understanding the adequacy of the score. For quality improvement and research comparisons, the AUROC is a useful single value in deciding whether a model can help determine differences in SOI between cohorts. ${ }^{13}$ For determining the predictive validity of a definition of sepsis, assessing mortality risk above baseline risk may be most appropriate. ${ }^{15}$ For deciding who needs escalation of care, the sensitivity and specificity with a particular cut-off value is likely to be more important in judging the adequacy of the model. ${ }^{11}$ Particularly in the latter example, which is the most often cited for scores in LMICs, care must be taken in how the scores are used for individual clinical decision-making since low sensitivity could lead to patients who need additional care being missed and low specificity could lead to attempts at using scarce resources for a relatively large population. ${ }^{112627}$

Our study has several strengths. We looked at adult patients across the entire hospital rather than the ICU alone, ${ }^{127101617}$ which is particularly important in settings where many critically ill patients remain outside the ICU due to limited ICU capacity. ${ }^{13}$ We also analysed the score performances in multiple ways: as continuous scores, continuous scores added to baseline risk and as dichotomous values. In addition, the retrospective multisite LMIC qSOFA validation included a cohort from the emergency department of our hospital; ${ }^{15}$ our cohort and that cohort showed similarly modest predictive capacity for the continuous qSOFA score without baseline model, providing criterion validity to our results (AUROC 0.55 in the multisite study and 0.65 in this study). Finally, other than one small study confined to emergency department patients and with a low $(5 \%)$ mortality rate, ${ }^{23}$ our study is the first to assess the UVA score outside of its LMIC derivation cohort. $^{13}$

Our study also has several limitations. First, we conducted it in a single tertiary care hospital in subSaharan Africa, so its results may not be generalisable. Even more complex SOI scores derived from much larger populations, such as the APACHE score for ICU patients in HICs, have quite variable performance, requiring recalibration for different populations and over time in the same population. ${ }^{122829}$ It is reasonable to expect that variations in patient characteristics, management systems, and resources across hospitals would translate to different predictive capacities of scores across hospitals. Of note, in the retrospective study of qSOFA in nine LMIC cohorts, the AUROC for all combined sites without the baseline model was 0.69 , but the AUROC range for individual sites was wide, from 0.55 to $0.81 .^{15}$ Second, the variables used to calculate the scores for patients in our study were recorded from different time points (time of fever, operation or culture sample retrieval) depending on the inclusion criteria each participant met for the study. This likely simulates how the scores might be used in practice; however, it is certainly possible the scores would perform better with more consistent data collection time points. We may also have a survivor bias of unknown direction since patients who died rapidly after admission to the hospital before they could be screened, or who died before infection was suspected, were not included. Third, oxygen saturation was included as a variable, without oxygen delivery; this was a feature of the UVA score design, but it nonetheless seems likely that oxygen saturation without oxygen delivery will be more limited in its predictive power. Fourth, we had some missing data, up 
to $11.7 \%$ for oxygen saturation, for which we assumed normal values; however, the missingness was relatively low compared with many other LMIC studies ${ }^{12}$ and reflects reasonable real world data availability. Fifth, our positive culture rate of $42.2 \%$ in this population is likely artificially high given that one of the inclusion criteria for the study was a positive culture. Finally, we were unable to evaluate the original MEWS score since we did not have detailed mental status data. We used an adapted MEWS with a binary version of the mental status variable without prior validation of this adaptation; these scores could have been overestimated or underestimated and, therefore, impacted the score's capacity to differentiate participants.

\section{CONCLUSION}

Our study found modest predictive power of adjusted MEWS, qSOFA and UVA scores in our cohort of inpatients with suspected infection at a Rwandan tertiary hospital. These modest predictive performances must be acknowledged if these scores are to be considered for use in research comparisons, quality improvement or clinical decision-making.

\section{Author affiliations}

${ }^{1}$ Department of Medicine, Beth Israel Deaconess Medical Center, Boston, Massachusetts, USA

${ }^{2}$ Harvard Medical School, Boston, Massachusetts, USA

${ }^{3}$ Department of Anesthesia, Critical Care, and Pain Medicine, Beth Israel Deaconess Medical Center, Boston, Massachusetts, USA

${ }^{4}$ Department of Surgery, Kigali University Teaching Hospital, Kigali, Rwanda ${ }^{5}$ University of Rwanda College of Medicine and Health Sciences, School of Medicine and Pharmacy, Kigali, Rwanda

${ }^{6}$ Division of Critical Care/Acute Care Surgery, Department of Surgery, University of Minnesota, Minneapolis, Minnesota, USA

${ }^{7}$ Division of Pulmonary, Critical Care, and Sleep Medicine, Department of Medicine, Beth Israel Deaconess Medical Center, Boston, Massachusetts, USA

Acknowledgements We thank Claude Mambo Muvunyi, Theoneste Nkubana, Emile Musoni and Jean-Paul Mvukiyehe for their excellent research assistance.

Contributors AK, AM, TS, CM, JR, DST and ER contributed to study conception and design, including methodology. Data acquisition was performed by TS, CM, EN, J-PN, ZN and JR. Analysis was performed by AM and ER. The first draft of the manuscript was written by AK and ER, and all authors commented on drafts of the manuscript. All authors read and approved the final manuscript. All authors agree to be accountable for all aspects of the work, including accuracy and integrity of the data and analysis.

Funding This work was supported by The Beth Israel Anesthesia Foundation and the University of Minnesota Department of Surgery.

Competing interests None declared.

Patient consent for publication Not required.

Provenance and peer review Not commissioned; externally peer-reviewed.

Data availability statement Data are available upon reasonable request. Deidentified data is available from the authors upon request.

Supplemental material This content has been supplied by the author(s). It has not been vetted by BMJ Publishing Group Limited (BMJ) and may not have been peer-reviewed. Any opinions or recommendations discussed are solely those of the author(s) and are not endorsed by BMJ. BMJ disclaims all liability and responsibility arising from any reliance placed on the content. Where the content includes any translated material, BMJ does not warrant the accuracy and reliability of the translations (including but not limited to local regulations, clinical guidelines, terminology, drug names and drug dosages), and is not responsible for any error and/or omissions arising from translation and adaptation or otherwise.
Open access This is an open access article distributed in accordance with the Creative Commons Attribution Non Commercial (CC BY-NC 4.0) license, which permits others to distribute, remix, adapt, build upon this work non-commercially, and license their derivative works on different terms, provided the original work is properly cited, appropriate credit is given, any changes made indicated, and the use is non-commercial. See: http://creativecommons.org/licenses/by-nc/4.0/.

ORCID iD

Elisabeth Riviello http://orcid.org/0000-0002-9443-3928

\section{REFERENCES}

1 Haniffa R, Isaam I, De Silva AP, et al. Performance of critical care prognostic scoring systems in low and middle-income countries: a systematic review. Crit Care 2018;22:18.

2 Lalani HS, Waweru-Siika W, Mwogi T, et al. Intensive care outcomes and mortality prediction at a national referral hospital in Western Kenya. Ann Am Thorac Soc 2018;15:1336-43.

3 Opio MO, Nansubuga G, Kellett J. Validation of the VitalPAC'TM early warning score (views) in acutely ill medical patients attending a resource-poor hospital in sub-Saharan Africa. Resuscitation 2013;84:743-6.

4 Aluisio AR, Garbern S, Wiskel T, et al. Mortality outcomes based on ED qSOFA score and HIV status in a developing low income country. Am J Emerg Med 2018;36:2010-9.

5 Khwannimit B, Bhurayanontachai R, Vattanavanit V. Comparison of the performance of SOFA, qSOFA and SIRS for predicting mortality and organ failure among sepsis patients admitted to the intensive care unit in a middle-income country. J Crit Care 2018;44:156-60.

6 Boillat-Blanco N, Mbarack Z, Samaka J, et al. Prognostic value of quickSOFA as a predictor of 28-day mortality among febrile adult patients presenting to emergency departments in Dar ES Salaam, Tanzania. PLoS One 2018;13:e0197982.

7 Sendagire C, Lipnick MS, Kizito S, et al. Feasibility of the modified sequential organ function assessment score in a resourceconstrained setting: a prospective observational study. BMC Anesthesiol 2017;17:12.

8 Carugati M, Zhang HL, Kilonzo KG, et al. Predicting mortality for adolescent and adult patients with fever in resource-limited settings. Am J Trop Med Hyg 2018;99:1246-54.

9 Baig MA, Sheikh S, Hussain E, et al. Comparison of qSOFA and SOFA score for predicting mortality in severe sepsis and septic shock patients in the emergency department of a low middle income country. Turk J Emerg Med 2018;18:148-51.

10 Prin M, Pan S, Kadyaudzu C, et al. Development of a Malawi intensive care mortality risk evaluation (mime) model, a prospective cohort study. Int J Surg 2018;60:60-6.

11 Machado FR, Cavalcanti AB, Monteiro MB, et al. Predictive accuracy of the quick sepsis-related organ failure assessment score in Brazil. A prospective multicenter study. Am J Respir Crit Care Med 2020;201:789-98.

12 Beane A, De Silva AP, De Silva N, et al. Evaluation of the feasibility and performance of early warning scores to identify patients at risk of adverse outcomes in a low-middle income country setting. BMJ Open 2018;8:e019387.

13 Moore CC, Hazard R, Saulters KJ, et al. Derivation and validation of a universal vital assessment (UVA) score: a tool for predicting mortality in adult hospitalised patients in sub-Saharan Africa. BMJ Glob Health 2017;2:e000344.

14 Baker T, Schell CO, Lugazia E, et al. Vital signs directed therapy: improving care in an intensive care unit in a low-income country. PLoS One 2015;10:e0144801.

15 Rudd KE, Seymour CW, Aluisio AR, et al. Association of the quick sequential (sepsis-related) organ failure assessment (qSOFA) score with excess hospital mortality in adults with suspected infection in low- and middle-income countries. JAMA 2018;319:2202-11.

16 Haniffa R, Mukaka M, Munasinghe SB, et al. Simplified prognostic model for critically ill patients in resource limited settings in South Asia. Crit Care 2017;21:250.

17 Riviello ED, Kiviri W, Fowler RA, et al. Predicting mortality in lowincome country ICUs: the Rwanda mortality probability model (RMPM). PLoS One 2016;11:e0155858.

18 Ranieri VM, Rubenfeld GD, et al, ARDS Definition Task Force. Acute respiratory distress syndrome: the Berlin definition. JAMA 2012;307:2526-33.

19 Singer M, Deutschman CS, Seymour CW, et al. The third International consensus definitions for sepsis and septic shock (Sepsis-3). JAMA 2016;315:801-10. 
20 Subbe CP, Kruger M, Rutherford P, et al. Validation of a modified early warning score in medical admissions. QJM 2001;94:521-6.

21 Morgan RJM WF, Wright MM. An early warning scoring system for detecting developing critical illness. Clincal Intensive Care 1997;8:100.

22 Kruisselbrink R, Kwizera A, Crowther M, et al. Modified early warning score (MEWS) identifies critical illness among ward patients in a resource restricted setting in Kampala, Uganda: a prospective observational study. PLoS One 2016;11:e0151408.

23 Schmedding M, Adegbite BR, Gould S, et al. A prospective comparison of quick sequential organ failure assessment, systemic inflammatory response syndrome criteria, universal vital assessment, and modified early warning score to predict mortality in patients with suspected infection in Gabon. Am J Trop Med Hyg 2019;100:202-8.

24 Sutherland T, Mpirimbanyi C, Nziyomaze E, et al. Widespread antimicrobial resistance among bacterial infections in a Rwandan referral hospital. PLoS One 2019;14:e0221121.
25 Seymour CW, Liu VX, Iwashyna TJ, et al. Assessment of clinical criteria for sepsis: for the third International consensus definitions for sepsis and septic shock (Sepsis-3). JAMA 2016;315:762-74.

26 Sinuff T, Adhikari NKJ, Cook DJ, et al. Mortality predictions in the intensive care unit: comparing physicians with scoring systems. Crit Care Med 2006;34:878-85.

27 Aoyama K, D'Souza R, Pinto R, et al. Risk prediction models for maternal mortality: a systematic review and meta-analysis. PLoS One 2018;13:e0208563.

28 Salluh JIF, Soares M. Icu severity of illness scores: APACHE, SAPs and MPM. Curr Opin Crit Care 2014;20:557-65.

29 Haniffa R, Beane A, Baker T, et al. Development and internal validation of the simplified mortality score for the intensive care unit (SMS-ICU). Acta Anaesthesiol Scand 2018;62:407-8. 